\title{
PARBATH: Aplicación móvil para localizar baños y estacionamientos
}

\section{PARBATH: Mobile application to locate bathrooms and parking lots}

\author{
Pablo Domínguez ${ }^{1}$, Samuel Sánchez ${ }^{1}$, Guelda Carballeda ${ }^{2 *}$ \\ ${ }^{1}$ Licenciatura en Desarrollo de Software - Centro Regional de Coclé - Universidad Tecnológica de Panamá \\ ${ }^{2}$ Facultad de Ingeniería de Sistemas Computacionales - Centro Regional de Coclé - Universidad Tecnológica de Panamá
}

Resumen En este artículo se presenta el desarrollo de la aplicación PARBATH, basada en el sistema operativo Android, la cual es una propuesta para localizar baños y estacionamientos públicos. La aplicación ha sido diseñada para ser utilizada por personas con discapacidad, incluyendo la discapacidad visual, ya que cuenta con diversas funciones de accesibilidad para su uso. No obstante, dada su versatilidad, también puede ser utilizada por otros usuarios que no tengan discapacidad. Adicionalmente, en este artículo, se hace una revisión breve de la literatura relacionada, la cual ha sido tomada como base para el desarrollo de la aplicación PARBATH. Finalmente, se hace una explicación detallada del funcionamiento de esta aplicación y se presenta los resultados experimentales obtenidos durante su validación.

Palabras clave Accesibilidad, android, baños, discapacidad visual, estacionamientos.

\begin{abstract}
This article presents the development of the PARBATH application, based on the Android operating system, which is a proposal to locate toilets and public parking lots. The application has been designed to be used by people with disabilities, including the visually impaired, since it has various accessibility functions for its use. However, given its versatility, it can also be used by other users who do not have a disability. Additionally, in this article, a brief review of the related literature is made, which has been taken as a basis for the development of the PARBATH application. Finally, a detailed explanation of the operation of this application is made and the experimental results obtained during its validation are presented.
\end{abstract}

Keywords Accessibility, android, bathrooms, visual impairment, parking.

* Corresponding author: guelda.carballeda@utp.ac.pa

\section{Introducción}

La discapacidad visual representa una limitación considerable para las personas que padecen esta condición. Al ritmo con el que avanza la tecnología, esta evoluciona, se adapta y abre paso a innumerables posibilidades para ayudar a quien no puede llevar una vida cotidiana normal; como es el caso de las personas con discapacidad visual (PcDV).

La Organización Panamericana de la Salud (OPS), reporta que en América Latina y el Caribe por cada millón de habitantes hay 5,000 personas invidentes y 20,000 personas con discapacidad visual. En cifras totales mundiales, hay alrededor de 269 millones de personas con discapacidad visual y 45 millones de invidentes. La ceguera parece ser más frecuente en el género femenino, puesto que los reportes señalan que cerca del $60 \%$ de la población invidente está conformado por mujeres. El $87 \%$ de las personas con discapacidad visual habitan países en desarrollo [1].
Según un estudio realizado por la Secretaría Nacional de Discapacidad de Panamá (SENADIS) [2], en Panamá por cada tres viviendas hay, al menos, una persona con algún tipo de discapacidad. Esto equivale a una tasa de $11.3 \%$, que representa a una población de 370,053 de personas con discapacidad (PcD) según la Primera Encuesta Nacional de Personas con Discapacidad (PENDIS), realizada en 2006. Se entiende que, actualmente, hay más personas con discapacidad, pero no se ha realizado otra encuesta de este tipo desde el año mencionado. Con relación a las personas con discapacidad visual ( $\mathrm{PcDV}$ ), el proceso para lograr cierta independencia para realizar sus actividades cotidianas es muy difícil y toma mucho tiempo para su entrenamiento, sin considerar el hecho de que existan otras limitantes que dificulten, aún más, alcanzar ese objetivo.

Este proyecto lleva por nombre: PARBATH (Sistema Localizador de Baños y Estacionamiento), el cual es una aplicación diseñada y desarrollada para teléfonos móviles 
basados en el Sistema Operativo (SO) Android desde la versión 4.4 y posteriores. Esta aplicación (app) pretende mejorar la calidad de vida de las PcDV y personas con algún otro tipo de discapacidad, ya que incorpora un motor de texto a voz y los mapas de navegación de Google Maps [3], con los servicios necesarios para trazar rutas, calcular distancias, localizar la posición del usuario, etc. Además, en el sistema propuesto se ha diseñado un prototipo con el uso de un módulo wifi esp8266, para procesar y enviar los datos obtenidos de un sensor ultrasónico a una base datos compartida con la aplicación, a fin de determinar, en tiempo real, si el baño o estacionamiento, localizado por la app, está libre u ocupado. Para lograr mostrar esta información a través de este prototipo se debe instalar los módulos wifi con su debido sensor en los lugares que se han identificado en la aplicación. En caso contrario, solo se indicará en la aplicación si el establecimiento o lugar posee baños o estacionamiento, pero sin entrar en detalle que esté libre u ocupado, en adición a otras características importantes para el conocimiento del usuario.

\section{Antecedentes}

Se han revisado algunos proyectos, modelos y propuestas, en la literatura científica, relacionados con el trabajo que se presenta en este artículo. Entre ellos, en [4] se encuentra un modelo orientado a objetos de representaciones enriquecidas, definidas como una representación espacial en interiores, que posee al menos una entrada, además de redes de circulación, con el fin de brindar la asistencia a los usuarios en la navegación en interiores y exteriores. Esta propuesta utiliza los sistemas de posicionamientos actuales, como Google Maps, que utiliza el GPS y, además, se basa en mapas generales y no brinda mapas internos. En el artículo presentado en [4] también se presenta un caso con ejemplos de instancia del modelo planteado.

Entre algunos proyectos, modelos y propuestas similare, encontramos un modelo orientado a objetos de representaciones enriquecidas, que posee al menos una entrada, además de redes de circulación, con el fin de brindar la asistencia a los usuarios en la navegación en interiores y exteriores, ya que los sistemas de posicionamientos actuales como Google Maps utiliza el GPS y además se basa en mapas generales y no brinda mapas internos. En el artículo presentado en [4] también se muestra caso de ejemplos de instancia del modelo planteado.

Otro proyecto interesante es el proyecto VIABLE (Entorno colaborativo, Tecnología web y Movilidad para la vida independiente y la accesibilidad) desarrollado por Treelogic, el Instituto Tecnológico de Informática y ONCE-CIDAT. Con VIABLE se ha desarrollado una plataforma inteligente basada en el concepto de red social, que es capaz de ofrecer información sobre las vías públicas y los posibles "puntos negros" de una ruta introducida. En caso de encontrar un punto, se considera si existe una ruta alternativa que no suponga un exceso innecesario de distancia a recorrer y que no tenga ninguna incidencia [5].

Por otro lado, en [6] se presenta una implementación de una aplicación móvil como solución viable para localizar a personas con discapacidad visual (total o parcial) dentro de recintos cerrados. Esta app utiliza el Bluetooth para conectar un bastón blanco, que permite la transmisión de información capturada por medio de la tecnología de Identificación por Radio Frecuencia (RFID) y una cinta magnética como guía [5].

\section{Descripción general}

Para el diseño y desarrollo de la aplicación PARBATH, se han utilizado las siguientes tecnologías:

- Google Maps API [7], utilizada para la integración del mapa de Google en la aplicación desarrollada,

- Google Maps Distance Matrix API [8], para determinar las distancias entre PcDV y los puntos de interés,

- Google Maps Directions API [8], para generar en el mapa las rutas más cortas al marcador seleccionado,

- Firebase [9], para almacenar la información de baños y estacionamientos,

- GPS [10], para determinar la posición de las PcDV,

- WIFI [11], para el acceso a internet y la interconexión inalámbrica de los dispositivos electrónicos y,

- Arduino [12], para determinar la disponibilidad de un baño, si se encuentra libre u ocupado.

Cada una de las tecnologías utilizadas, enumeradas anteriormente, es indispensable para el correcto funcionamiento del sistema desarrollado. En cuanto a la accesibilidad, para las personas con discapacidad visual se implementó un motor de texto a voz en la aplicación mediante la plataforma de desarrollo Android Studio, además de contar con interfaces con listas que resumen la información que se desea presentar.

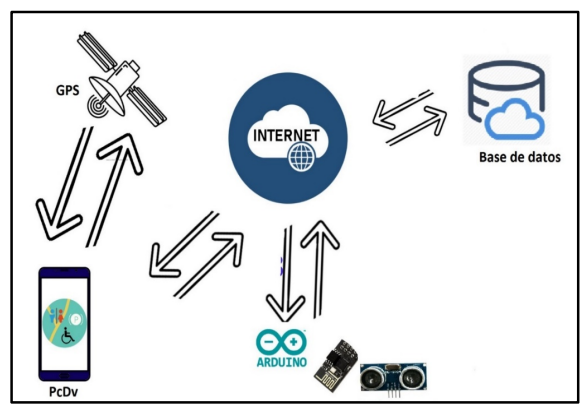

Figura 1. Modelo conceptual de la aplicación.

El modelo conceptual de PARBATH, como se muestra en la figura 1, lo inicia el usuario con un teléfono inteligente que cuente con el sistema operativo Android. La navegación se realiza por medio del GPS, la disponibilidad de baños y estacionamientos depende del módulo sensorial de proximidad 
y del módulo WIFI, ambos programables en el entorno de desarrollo Arduino.

Con el uso de la aplicación y servicios de Google, se permite la movilidad de personas en ambientes abiertos. Cuando una PcDV requiere obtener información sobre la localización de baños y estacionamientos, ella inicia la utilización de la app PATBATH y por medio del motor de texto voz, se brinda información sobre lo que está en la interfaz de la app. Para ello, sobre la interfaz de mapa de la app selecciona un marcador, que contiene la información de un baño o estacionamiento, y el sistema le responde con la distancia aproximada y la ruta entre el usuario y el destino seleccionado. Adicionalmente a lo anterior, el usuario podrá encontrar lugares de interés, dentro del entorno de la ruta seleccionada, de una lista dinámica que está implementada en el sistema, proporcionando, además, la distancia aproximada a su posición. Para conocer más detalles sobre el punto de interés elegido solo basta seleccionarlo y se mostrará información específica: horario, disponibilidad y valoración. Todo esto, junto con los marcadores del mapa se encuentra alojada en una base de datos (véase la figura 1).

\section{Desarrollo del sistema}

PARBATH proporciona información de baños y estacionamientos como: ubicación, descripción del lugar, disponibilidad, comentarios de otros usuarios, horarios. Mediante las consultas realizadas a las APIs de Google se logra mostrar la distancia hacia un lugar, además mostrar la ubicación actual. Para que la aplicación fuera más accesible para las PcDV se optó por enseñar la información del mapa en listas, en una forma resumida, con nombre del lugar y de la distancia a la que se encuentra, además de contar con mensajes audibles.

La figura 2, 3 y 4 son muestra de algunas interfaces que se han diseñado para lograr la navegabilidad de las personas, cada una con funciones auditivas para el usuario con discapacidad visual.

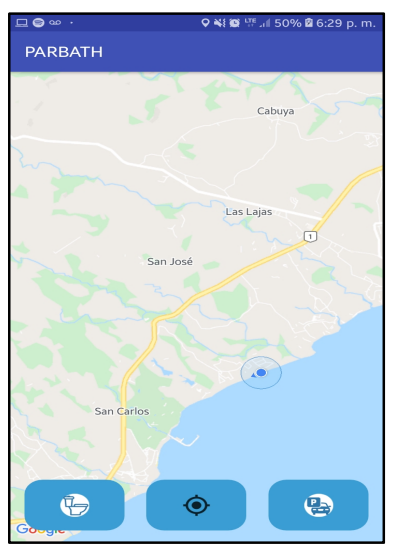

Figura 2. Interfaz ubicación.
La interfaz "Ubicación" (ver figura 2) es la primera que aparece cuando se abre la aplicación PARBATH. Esta será la primera en ejecutarse, cuenta con un mensaje audible que le indicará a la PcDV lo que puede hacer para poder utilizar la aplicación. Entre las indicaciones, se le informa que existen botones en la parte inferior, que el de la izquierda y derecha son para determinar el baño o estacionamiento más cercano y el botón del centro determinará la posición actual de la PcDV.

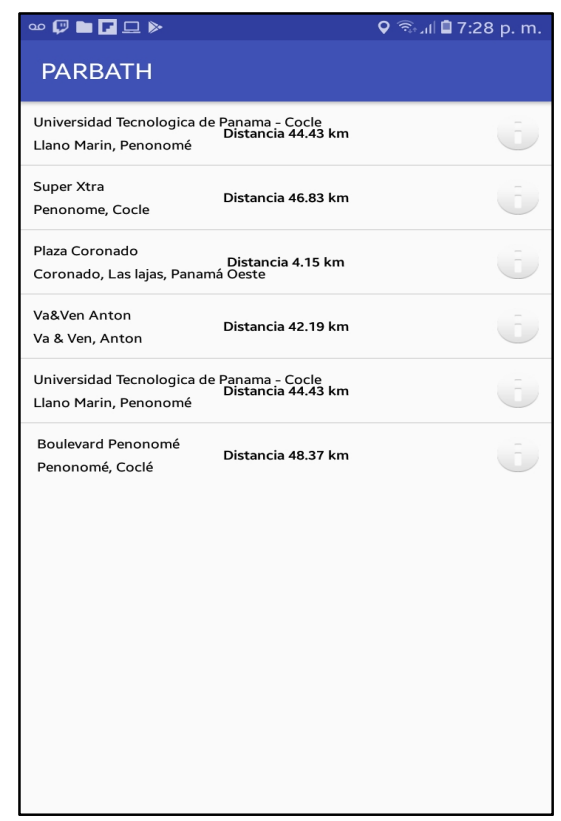

Figura 3. Interfaz alternativa mapa.

Con el mensaje también se le indicará que, girando el celular a la derecha, podrá acceder a la interfaz "Alternativa Mapa" (véase figura 3), que fue creada con el objetivo de mostrar los lugares registrados en el mapa en una lista de forma sencilla y con información de la distancia aproximada a la que se encuentra cada uno de estos lugares.

En la interfaz de ubicación de baño o estacionamiento, al seleccionar un marcador, se mostrará la ruta más corta para llegar al destino elegido, además en una pestaña en la parte inferior, la distancia y el nombre del lugar. Al seleccionar un lugar de lista "Alternativa mapa", podrá acceder a la interfaz lista servicio donde se muestra un listado de baños y estacionamientos con los que cuenta el local o centro comercial. Al seleccionar un elemento de la lista, se accede a la Interfaz "Información baño o estacionamiento" (ver figura 4) que contiene la descripción del lugar, horario, disponibilidad, comentarios e información extra que puede ser de ayuda.

Al presionar el botón de reporte, se tendrá acceso al interfaz formulario, esta última es donde el cliente (PcDV) podrá realizar un comentario o dejar su opinión de la experiencia que 
ha tenido durante su estancia en ese lugar, la cual servirá como una opinión de ayuda para otras personas que quieran acudir a ese sitio.

Para lograr mostrar la disponibilidad de baños en la aplicación, se realizó la investigación de algunos proyectos de Arduino que ayudaron a alcanzar este objetivo. Entre los proyectos revisados se encuentran: un circuito con sensor HCSR04 [12], sensor infrarrojo HC-SR501 con Arduino [13]. Sin embargo, estos proyectos no contaban con una base de datos para el envío de la información registrada por los sensores. Ahora bien, para comunicar los datos de los sensores de ultrasonido e infrarrojos, se integró al sistema PARBATH el módulo WIFI ESP8266 [14,15], el cual puede conectarse a una red WIFI específica, establecida en el código del sistema, y con ello se logra comunicar los resultados con la aplicación.

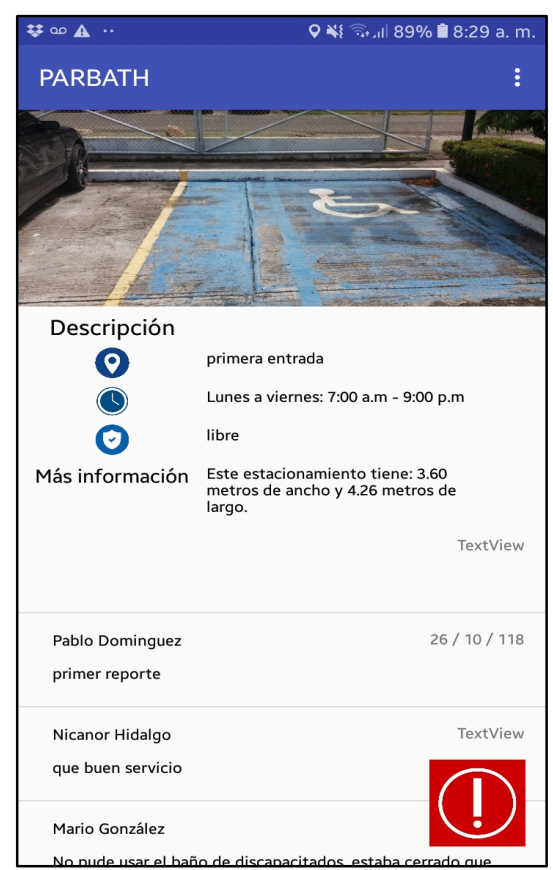

Figura 4. Interfaz Información baño o estacionamiento.

En la figura 5 se ve el diagrama de un prototipo, con diferentes componentes de Arduino para mostrar la disponibilidad de un baño en un lugar seleccionado. Los componentes utilizados son los siguientes: módulo WIFI ESP8266, sensor ultrasónico (también puede utilizarse un sensor infrarrojos), placa de 400 pines, fuente de poder con salida de 3.3 y 5 voltios, y batería de 9 voltios.

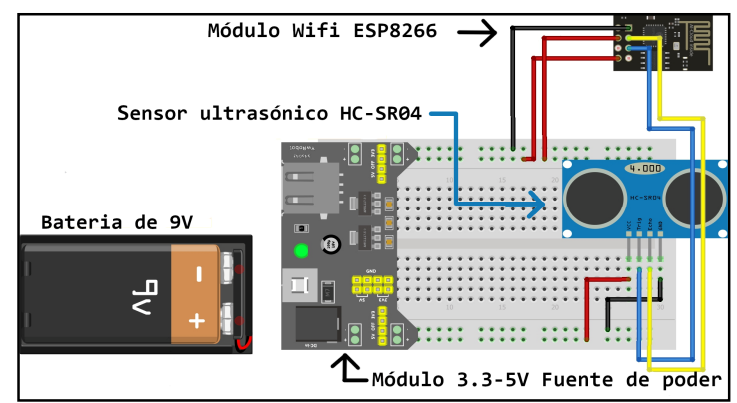

Figura 5. Sub-sistema sensorial ultrasónico compatible con Módulo Wifi ESP8266.

Este prototipo puede ser colocado en un baño o estacionamiento para lograr enviar la información y cumplir con su objetivo. Sin embargo, se debe adecuar dentro de una caja específica para su buen funcionamiento y mientras este no sea instalado, solo se mostrará la cantidad de baños y estacionamientos que posea el lugar elegido en la app. Con el sensor ultrasónico se podrá detectar si hay alguna persona utilizándolo. En este caso, el sensor envía una señal de detección al módulo WIFI, el cual debe estar conectado a una red inalámbrica, para enviar el dato pertinente a la base de datos (Firebase), y desde allí se envía la información a la app que porta la PcDV, indicando que el baño se encuentra ocupado. Los códigos implementados en el sistema PARBATH, esto es, en la app como en los módulos electrónicos de detección y WIFI, están diseñados para que se ejecuten en tiempo real, para que, en este caso, la PcDV pueda saber si el baño está ocupado o desocupado, dependiendo del momento específico.

\section{Pruebas experimentales}

Una primera prueba de campo fue realizada en la Universidad de Panamá, Centro Regional Universitario de Coclé, en conjunto con Asuntos Estudiantiles se nos facilitó enviarle una carta, a la directora del centro, comentándole sobre la iniciativa y las pruebas que necesitábamos hacerles a las personas con discapacidad dentro del mismo. La directora Betty J Gómez, nos respondió con éxito nuestra solicitud para proceder. También, la estudiante Katerina Taylor (ver Figura 6) a través de una entrevista nos brindó su consentimiento, para colaborar con el proyecto probando la aplicación. En la entrevista se plantearon varias preguntas con respecto a su condición. Finalmente su firma y cédula para proceder con la prueba. Seguido la aplicación fue instalada en su teléfono Samsung Galaxy J5 Prime y no hubo ningún tipo de incompatibilidad con su modelo de celular. La app se instaló correctamente. 


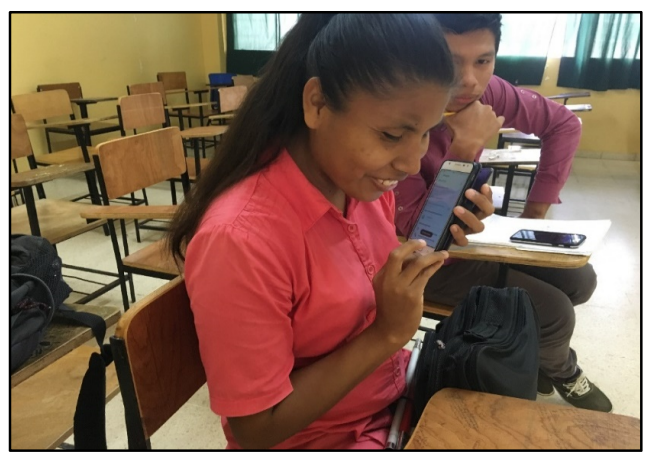

Figura 6. Detalle inicial de una prueba de campo.

Para el resumen de la prueba sobre la experiencia con el uso de la aplicación, al inicio fue complicado el acceso a la app, debido a los permisos de ubicación que la aplicación solicita para poder utilizar el GPS. Por tanto, la estudiante logró navegar entre las diferentes interfaces, desconociendo el uso de la app con los pocos minutos de uso, obtuvo información necesaria para localizar uno de los baños que eligió. Sin embargo, hay aspectos que se podrían mejorar, como la manera de cómo se indica la distancia al elegir un baño o estacionamiento, que existieran puntos de referencia conocidos, y que se agregaran lugares de interés. Finalmente, la joven añade que ninguna aplicación será fácil de manejar para ellos por primera vez, pero todo es cuestión de ir probando y adaptándose a la mejor manera de utilizarla.

Según los resultados de la prueba realizada, se procedió a efectuar mejoras a la aplicación para facilitar su uso.

Las mejoras realizadas son las siguientes:

- Implementación de navegación por listas como alternativa al mapa de navegación, ya que este puede resultar complicado de usar para una PcDV,

- Nuevos mensajes audibles que ayudarán al uso de la aplicación, y

- Modificación de las interfaces, para una mejor interacción con el usuario.

Una segunda prueba de campo fue realizada después de hacer las mejoras al sistema comentadas anteriormente. En ella no hubo problemas de instalación ni de acceso a la aplicación. Sin embargo, la Srta. Taylor sugirió que, una vez dentro de la app, el usuario reciba una guía rápida sobre acciones que puede realizar o atajos, por ejemplo, el uso de la interfaz alternativa al mapa, indicando que para acceder a ella solo basta girar el teléfono hacia la derecha. En comparación con la primera prueba, ella afirmó que hubo una gran mejora en las indicaciones de orientación hacia un baño o estacionamiento, lo cual es fundamental para su movilización adecuada, ya sea que vaya sola o acompañada.

En las pruebas se observó que algunos de los mensajes audibles que se establecieron en la aplicación son omitidos por el sistema, ya que entra en conflicto con el narrador del sistema
(Talkback) y este último es el que posee prioridad sobre los mensajes que se desean transmitir.

\section{Conclusiones}

El prototipo presentado en este trabajo está orientado a funcionar, en espacios abiertos por el uso de la tecnología GPS y la información que se muestra de las peticiones a las APIs de Google. Con esta iniciativa de estudio de caso, se logra presentar a las personas, y también para aquellas con alguna discapacidad, una nueva opción para localizar los diversos puntos en donde puedan suplir sus necesidades básicas frente al desconocimiento que existe en la actualidad, al no contar con un sistema o protocolo que los ayude a conocer dicha información. A pesar de que se logra el objetivo planteado, se espera que con la opinión de las PcDV se puedan tomar en cuenta otras adaptaciones que podrían mejorar la aplicación e incluso la unión con otros sistemas para la movilidad en interiores y facilitar mucho más el desplazamiento en lugares cerrados.

La aplicación PARBATH, descrita en este artículo, forma parte de un proyecto investigación y desarrollo que lleva como título "Diseño e implementación de sistemas basados en las TIC para ayudas en la movilidad de personas con discapacidad visual en interiores para favorecer su inclusión social", cuyo acrónimo es MOVIDIS-II. Esto indica que PARBATH ha sido desarrollado considerando los parámetros metodológicos científicos que todo proyecto de I+D requiere. En [16] y [17] se presenta una descripción ampliada del proyecto MOVIDISII.

\section{AGRADECIMIENTOS}

Este trabajo ha sido realizado dentro del marco del proyecto MOVIDIS-II financiado por la SENACYT (Programa FID2017) con Contrato por Mérito No: 99-2018-4-FID17-031. Agradecemos al Dr. Héctor Montes, IP del proyecto MOVIDIS-II, por la revisión de este artículo y las recomendaciones dadas.

\section{REFERENCIAS}

[1] Ministerio de Salud de Panamá MINSA. (2011, Jun). "Recomendaciones para facilitar la integración de las Personas con Discapacidad Visual”. Boletín N5. [En línea]. Disponible en:

http://www.minsa.gob.pa/sites/default/files/publicaciones/bolet in_5_discapacidad_visual.pdf [Consultado: 02-mayo-2020]

[2] Secretaría Nacional de Discapacidad SENADIS. (2006, Oct). "Estudio sobre la prevalencia y caracterización de la discapacidad en la República de Panamá". [En línea]. Disponible

http://www.senadis.gob.pa/documentos/vitacora/informependis.pdf [Consultado: 02-mayo-2020]

[3] P. D. P. J. Abhishek Shakwala, "Location Based Services and Integration of Google Maps in Android", int. jour. eng. com. sci, vol. 3, no. 03, Mar. 2014. [En línea]. Disponible en: 
http://103.53.42.157/index.php/ijecs/article/view/190 [Consultado: 23-jun-2020]

[4] A. B. Lliteras, C. Challiol, C. A. Mostaccio, S. E. Gordillo. (2011). "Representaciones enriquecidas para la navegación indoor-outdoor en aplicaciones móviles", in XVII Congreso Argentino de Ciencias de la Computación, 2011, pp. 867-876. [En línea]. Disponible en: http://hdl.handle.net/10915/18743 [Consultado: 06-may-2020]

[5] J. A. Muñoz Sevilla. (2012) "Las TIC y la discapacidad visual". [En línea]. Disponible en: http://hdl.handle.net/2183/13227 [Consultado: 06-may-2020]

[6] A. A. González Gómez, L. E. Pallares M., y R. Ferro Escobar, «Implementación de un dispositivo basado en Bluetooth y RFID para guía y posicionamiento en interiores de personas con incapacidad visual usando una APP en su dispositivo móvil.», redes ing., pp. 97-106, 1. [En línea]. Disponible en: https://doi.org/10.14483/2248762X.11996 [Consultado: 08may-2020]

[7] F. Wang, Y. Xu. (2011, Nov). "Estimating O-D travel time matrix by Google Maps API: implementation, advantages, and implications". [En línea]. Disponible en: https://doi.org/10.1080/19475683.2011.625977 [Consultado: 08-may-2020]

[8] P. D. P. J. Abhishek Shakwala, "Location Based Services and Integration of Google Maps in Android", int. jour. eng. com. sci, vol. 3, no. 03, Mar. 2014. [En línea]. Disponible en: http://www.ijecs.in/index.php/ijecs/article/view/190 [Consultado: 10-may-2020]

[9] A. R. Wiratno, K. Hastuti, "Implementation of Firebase Realtime Database to Track BRT Trans Semarang", Scientific Journal of Informatics, 2017, 4(2), 95-103. [En línea]. Disponible en: https://doi.org/10.15294/sji.v4i2.10829 [Consultado: 08-may-2020]

[10] O. Jiménez. (2019, Jul). "Geoposicionamiento en interiores". [En línea]. Disponible en: https://ruidera.uclm.es/xmlui/bitstream/handle/10578/23131/T FG_OscarJimenezJimenez.pdf?sequence $=1 \&$ isAllowed $=\mathrm{y}$ [Consultado: 23-jun-2020]

[11] R. Osorio, D. Duran, P. González M. Peña, G. Lefranc. (2010). "Geolocalización usando Tecnología WiFi (Wireless Fidelity)" in In Congreso Latinoamericano de Automática-Congreso ACCA. [En línea]. Disponible en: http://mediteka.iimas.unam.mx/web/lineasInv/pdf/WIFI-GEO LCA_RomanL Lefranc 2010.pdf [Consultado: 23-jun-2020]

[12] J. Cana. (2016, En) "Sensor HC-SR04 para crear una alarma con Arduino", HETPRO. [En línea]. Disponible en: https://hetprostore.com/TUTORIALES/sensor-hc-sr04/ [Consultado: 17may-2020]

[13] V. García. (2017, Nov) "Sensor HC-SR501 con Arduino." [En línea]. Disponible https://www.diarioelectronicohoy.com/blog/sensor-hc-sr501con-arduino [Consultado: 17-may-2020]

[14] L. Hernández, "ESP8266 todo lo que necesitas saber del módulo WiFi para Arduino" Programación fácil. [En línea]. Disponible en: https://programarfacil.com/podcast/esp8266-wifi-costearduino/ [Consultado: 17-may-2020]

[15] V. Ventura. (2015, Feb) "Conexión WiFi con un módulo ESP8266”. Polaridad. [En línea]. Disponible en: https://polaridad.es/modulo-wifi-esp8266/ [Consultado: 17may-2020]
[16] H. Montes, Design of a system to support the mobility of visually impaired people, congresoutp, vol. 1, n. ${ }^{\circ}$ 1, pp. 37-44, nov. 2018. [En línea]. Disponible en: https://revistas.utp.ac.pa/index.php/memoutp/article/view/1874 [Consultado: 05-jun-2020]

[17] H. Montes, I. Chang, G. Carballeda, J. Muñoz, Y. Saez, R. Vejarano, A. Garcia, "Conceptual design of technological systems for the mobility of visual impairment people in indoor buildings", in Proc. 7th International Engineering, Sciences and Technology Conference (IESTEC), Panama, 2019, pp. 1-6. [En línea]. Disponible en: https://ieeexplore.ieee.org/document/8943749 [Consultado: 05-jun-2020] 